\title{
Study on the Design and Function of Score Management System of Student Physical Health Test
}

\author{
Miao Zhong \\ Hunan Vocational College of Modern Logistics, Changsha, Hunan, 410131
}

Keywords: student physical health test; score management; design and function

\begin{abstract}
Student physical health test is an important part of middle school teaching management and an important indicator of school assessment. Student physical health test has always been a heavy-duty, time-sensitive, error-prone and confidential business. At present, because of the lack of technical means, the management of the physical assessment of middle school students still adopts a pure manual method, which is that the results data of physical measurements is exported into a format. Although this operation is simple, it cannot provide real-time query, subject to the disadvantages of manual management with the inflexible operation and the high error rate. More importantly, there is no way to provide data statistics, performance analysis, and other functions. In order to be able to change this situation, to better meet the needs of school development, and to reduce the work pressure of teachers, it is imminent to integrate seamlessly the national student physical health data reporting software, and to develop a physical health assessment management system suitable for the actual situation of middle school students. Therefore, it is necessary to design and implement a physical assessment score management system for middle school students.
\end{abstract}

\section{Introduction}

In recent years, the country has paid more and more attention to the physical fitness of middle school students. Sports performance has become an important part of the examination. In some provinces and cities, sports performance has been part of the college entrance examination. In middle school teaching, physical education is an important part. In the teaching management of middle schools, the management of student achievement has always been a heavy workload. As a part of student achievement, sports performance management has its own particularity. Compared with the scores of other courses, the scores of physical education courses consist of three parts, namely the scores in class, the results of physical education assessment, and the results of physical fitness assessment. At present, because of the lack of technical means, the management of the physical assessment of middle school students still adopts a pure manual method, that is, after the students have completed their physical measurements, they will export and export the results data into a format. Although this operation is simple, it can not provide real-time query, and is subject to the disadvantages of manual management, the operation is not flexible enough, and the error rate is relatively high. More importantly, there is no way to provide data statistics, performance analysis, and other functions. In order to be able to change this situation, in order to better meet the needs of school development, reduce the work pressure of classroom teachers; better integrate seamlessly with the national student physical health data reporting software, and publish the physical fitness score management suitable for the actual situation of middle school students. The system is imminent. Therefore, it is now decided to design and implement a physical assessment score management system for middle school students, and to carry out rational function design and page beautification for the system, hoping to build a simple, friendly, flexible and practical, safe and reliable physical fitness assessment score for secondary schools. Management system [1].

\section{Analysis of System Requirements}

In the process of physical education in middle school, the entry of sports results is a major task for middle school PE teachers. The results of physical fitness tests are divided into multiple 
categories and multiple categories. The data is more tedious and error-prone. The purpose of the system for developing physical examination of middle school students is to manage students easily. Physical performance test data, improve the performance of physical education teachers to enter the work efficiency, improve the accuracy of the input, free the physical education teacher from the boring work, and apply the limited time to improve the quality of physical education. The system's needs are obtained according to the physical education teacher's entry in the student's physical test score data. The daily work of physical test score management includes: input and management of physical health test data, student's daily performance management, student's class performance and attendance. Management and so on. The work includes statistics on student sports scores, statistics by class, and comprehensive results by project. These statistical requirements are presented in the form of charts and tables and need to be able to print. Based on the above work, the requirements involved in the system also include: student information management, teacher information management, class information management, and sports related data dictionary management [2]

\section{Content of Physical Monitoring}

The indicators of health assessment are divided into three categories. There are 19 basic indicators in fitness determination, 15 derived indicators, and 8 health checkups. Formulate evaluation criteria for quality indicators of various age groups, and the software can realize automatic calculation and evaluation. (1) The basic indicators include: (1) pulse, (2) systolic pressure, (3) diastolic pressure (Variation), (4) diastolic pressure (silence), (5) vital capacity, (6) height, (7) sitting height, (8) weight, (9) chest circumference, (10) shoulder width, (11) pelvic width, (12) standing long jump, (13) pull-up, (14), grip (15) step test (16) Sitting body flexion, (17) One-minute sit-ups (18) 800 meters, (19) 1000 meters. (2) Derivative indicators. Specifically include: (1) (height/height) × 100, (2) (body weight/height) $\times 100$, (3) (bath/height) $\times 100$, (4) (height/height) $\times 100$, (5) (pelvis width / height) $\times 100$, (6) [(weight + chest circumference) $/$ height] $\times$ 100, (7) weight - (height -100), (8) [(systolic pressure - diastolic pressure (various sound )) / Systolic blood pressure $\times 100$, (11) [(systolic pressure - diastolic pressure (silence) / systolic blood pressure) $\times 100$, (12) vital capacity / weight, (13) vital capacity / height, (14) vital capacity / chest circumference $(15)$ Weight $\times$ height $\times 0.43 \times$ pull-ups $/ 100$.

(3) Health indicators. Including vision, trachoma, heart, lungs, liver and spleen, neurasthenia, dental caries, scoliosis check.

College students' fitness measurement software quantifies the physical characteristics, loss of movement, and pathological features of the testers, and studies the relationship between measurement data and physical health. To quantify, integrate, and correlate many factors that reflect physical fitness, establish a database-based data model, classify and monitor physical fitness monitoring information, and share information through the Internet, so as to improve the efficiency of college student physique monitoring [3].

The database is the carrier for storing all information. The database of this system is divided into three module databases: basic information database, measurement data repository, and measurement standard comparison database. (1) Basic information database: Store basic archives data, basic student information, and tester basic information. (2) Measurement data repository: record all measurement information, including the measurement item's score, score, etc.

(3) Measurement standard comparison database: record the normal standards of various indicators set by the World Health Organization, and use it to compare with the scores of the measured persons, so as to obtain the performance level and physical fitness level of the measured persons.

\section{Description of System Software Structure and Module}

According to the demand, the university student physique monitoring and evaluation system can use two-dimensional function maps, represented as horizontal three business working layers: information transaction layer, information transaction analysis layer, statistical information decision 
layer, and vertical three operation presentation layers: Information Management System, Information statistics system, recommendation generation system system is divided into entry system, statistical system, evaluation system, recommendation system, management system, query system. (1) Entry system: to connect physical information with massive databases, and enter physical information including 19 basic indicators, 15 derived indicators, and 8 health check items. And set up the data processing subsystems of the above three indicators respectively, (2) Statistical system: Through the statistics of physical data, generate grade statistics, class statistics, school statistics (3) Assessment system: through the basis of each age group The correlation coefficient between indicators and derived indicators compares personal physical information with monitoring standards and generates an assessment of individual physique based on data analysis. (4) Suggestion System: Generate adaptive exercise prescriptions and fitness guidance. (5) Management System: Log in with the administrator password. Administrators can implement software upgrades, data entry, modification, monitoring, and screening. (6) Inquiry system: The monitored students can use the software to use the software to implement personal fitness query, evaluation and related suggestions [4].

According to the characteristics of physical fitness monitoring in colleges and universities, the database system is used as the bottom end, through the $\mathrm{B} / \mathrm{S} \& \mathrm{C} / \mathrm{S}$ multi-layer architecture, the interface of different interfaces is realized and the function is realized. Functionally, it can be divided into four major systems: college physique monitoring platform, basic information management system, external information system, and data statistics output system.

Consistency item information management system: The item for physical fitness measurement is the preparation before physical fitness measurement. The system records the name, number, category, measurement unit, measuring instrument, measurement site and other information of all items. The system administrator can view it at any time. , modify, delete, and add new items. Must-measure project information management system: Take the height, weight, and vital capacity of basic measurement items as fixed test items, other endurance items, flexible strength items, and speed smart items. Physical fitness monitoring project to measure. Physical measurement score entry system: Automatically identify basic information such as the student's name, grade, and major, and enter the score and score of the entered person. Physical fitness measurement management system: Complete modification, deletion, and re-addition after physical information input. Constitution measurement inquiry system: Through this system, students can log in to the system network terminal to inquire about their own constitution information, and obtain constitution analysis and exercise suggestions.

Physical information collection system: The latest information of physical monitoring is collected, organized, stored, and assisted software management personnel to perform necessary database updates and software upgrades. Information expansion system: The use of websites and other forms to achieve contact with local colleges and sports committees, timely dissemination of useful information obtained by the school's physique monitoring, and networking and opening of national scientific research institutes and national physique systems. Micro-statistics: Analyze the physical functions of the students who are measured and the movement of each part according to their athletic performance, so as to give the exercise suggestions and plans of the students to be measured. Macro-statistics: Statistics on physical information are used to generate grade statistics, class statistics, and school statistics [5].

\section{Conclusion}

The system makes full use of the existing IT technology, combines the development of the education system and further deepens the management needs, provides an accurate and efficient platform for the management of high school sports scores, and can maximize cost savings, improve economic efficiency and work efficiency. During the system bursting process, a system with good scalability and maintainability was implemented to meet the long-term development goals of the system, and the scalability and ease of use of the system were continuously improved. After the system is finally implemented, it will be successfully run in a secondary school, which will increase 
the efficiency and accuracy of physical education teachers, liberate them from cumbersome and tedious work, and improve the management level.

\section{Acknowledgements}

Hunan Provincial Department of Education Science Research Project (15C0964)

\section{References}

[1] Chen Yalin. Development and Application of "Student Physical Health Test Management" Software [J]. Sports Expo. 2011 (19)

[2] Lü Guanyan, Li Fenhua. UML-based Information System Requirements Analysis Model [J]. Microcomputer and Application. 2010 (20)

[3] Chen Shuli, mother Chenchen, Zhang Shugui. Research and design of student management information system based on Struts and Hibernate [J]. Software Guide. 2010 (07)

[4] Han Li. "SQL Server Database Fundamentals" Course Teaching Practice - For Computer Application Majors in Higher Vocational Colleges [J]. Science, Education, Culture, and Literature (late edition). 2010 (05)

[5] Wang Ge. A study on scores of general sports in colleges and universities [J]. Science and Technology Information. 2010 (12)

[6] Wang Qicai. Design and implementation of college sports performance management system [J]. Office Automation. 2010 (08) 